PAPER

\title{
Clinical significance of detection of multiple acute brain infarcts on diffusion weighted magnetic resonance imaging
}

\author{
V Caso, K Budak, D Georgiadis, B Schuknecht, R W Baumgartner
}

J Neurol Neurosurg Psychiatry 2005;76:514-518. doi: 10.1136/jnnp.2004.046383

See end of article for authors' affiliations

.....................

Correspondence to: Professor R W

Baumgartner, Department of Neurology, University Hospital of Zurich, Zurich 8091, Switzerland; ralf.baumgartner@ nos.usz.ch

Received 25 May 2004 Revised version received 15 July 2004

Accepted 22 July 2004
Background: Detection of multiple acute brain infarcts (MABI) by diffusion weighted magnetic resonance imaging (DWI) may provide information about stroke mechanism in (1) acute lacunar stroke, where evidence of $M A B I$ suggests a cause other than small artery disease (SAD), such as embolism or vasculitis (type $1 \mathrm{MABI}$ ); or (2) acute non-lacunar stroke, where MABI in the territory of at least two of the aortic branches supplying the brain indicates the presence of aortic or cardiac embolism rather than artery to artery embolism (type $2 \mathrm{MABI}$ ).

Objective: To evaluate the prevalence of $M A B I$ and their impact on aetiological classification and prevention of stroke in patients with acute ischaemic stroke examined with DWI.

Methods: 182 consecutive patients defined by DWI were evaluated. Stroke aetiology was classified according to the TOAST criteria, though "lacunar stroke" included patients with possible aetiologies other than SAD.

Results: Type $1 \mathrm{MABI}$ were detected in 21/72 patients (29\%) with lacunar stroke, and type $2 \mathrm{MABI}$ in 8 / $110(7 \%)$ with non-lacunar stroke. A possible stroke mechanism different from SAD was found in nine type $1 \mathrm{MABI}$ cases (43\%): cardiac embolism (4); other determined aetiology (3); aortic embolism (2). Cardiac (2) or aortic (1) sources of embolism were detected in eight type $2 \mathrm{MABI}$ cases. MABI patients with cardiac or aortic sources of embolism were treated with warfarin, the remainder with aspirin.

Conclusions: Detection of type $1 \mathrm{MABI}$ in patients with lacunar stroke improved diagnostic confidence and the choice of antithrombotic treatment. Further study is needed on stroke prevention in MABI cases caused by SAD alone.
D iffusion weighted magnetic resonance imaging (DWI) detects acute ischaemic infarcts of the brain within the first hour after the onset of symptoms and allows differentiation between acute and chronic lesions. ${ }^{1-3}$ Apart from identifying the acute symptomatic lesion, DWI may reveal multiple acute brain infarcts (MABI). ${ }^{4}$ Such a finding is of particular interest in two circumstances: first, in patients with acute ischaemic lacunar stroke the detection of MABI suggests the presence of a stroke mechanism other than small artery disease (SAD) — such as embolism, vasculitis, or coagulopathy (type 1 MABI; fig 1); second, in patients with acute non-ischaemic lacunar stroke, DWI evidence of MABI in the territory of two aortic branches suggests that aortic or cardiac but not arterial embolism is the mechanism of stroke (type 2 MABI; fig 2). Thus detection of MABI may improve both our knowledge of the aetiology of stroke and the preventive strategies.

We examined the prevalence of both MABI types in consecutive patients with first ever acute ischaemic stroke who underwent DWI of the brain, and evaluated their impact on the determination of the stroke aetiology and prevention.

\section{METHODS}

The Zürich ischaemic stroke registry has collected data prospectively from all patients admitted with a first ischaemic stroke (focal neurological deficit lasting $\geqslant 24$ hours) to the department of neurology of the University Hospital of Zürich since August 1997.5 In this paper we present an analysis of all inpatients who arrived within seven days after the onset of stroke symptoms, underwent DWI at the discretion of the treating physician, and were admitted between September 1999 and October 2001. During the same period, 232 inpatients and 205 outpatients with first ever stroke were registered.
A type 1 MABI was diagnosed if a patient with an acute lacunar stroke showed one or more other acute lacunar or non-lacunar brain infarcts on DWI. A type 2 MABI was diagnosed if a patient with acute non-lacunar stroke showed one or more further acute brain infarcts in the territory of another branch of the aorta. Consequently, type 2 MABI may be localised in the territories of the brachiocephalic trunk and the left common carotid artery, the brachiocephalic trunk and the left subclavian artery, or the left common carotid and the left subclavian arteries.

Patients who were being treated with intravenous or intra-arterial thrombolysis, who were included in studies evaluating glycoprotein IIb/IIIa receptor inhibitors or neuroprotective drugs, or who had suffered a iatrogenic stroke because of diagnostic or therapeutic interventions were excluded from the study.

\section{Investigations}

Information on the following stroke risk factors was obtained:

- age;

- sex;

- current cigarette smoking (including by definition cigarette smoking within the last five years);

- former cigarette smoking (defined as abstention from cigarette smoking for more than five years ${ }^{6}$ );

- hypertension, defined by preadmission history and medical records; diabetes mellitus, defined by past medical

Abbreviations: $A D C$, apparent diffusion coefficient; DWI, diffusion weighted magnetic resonance imaging; $\mathrm{MABI}$, multiple acute brain infarcts; NIHSS, National Institutes of Health stroke scale 

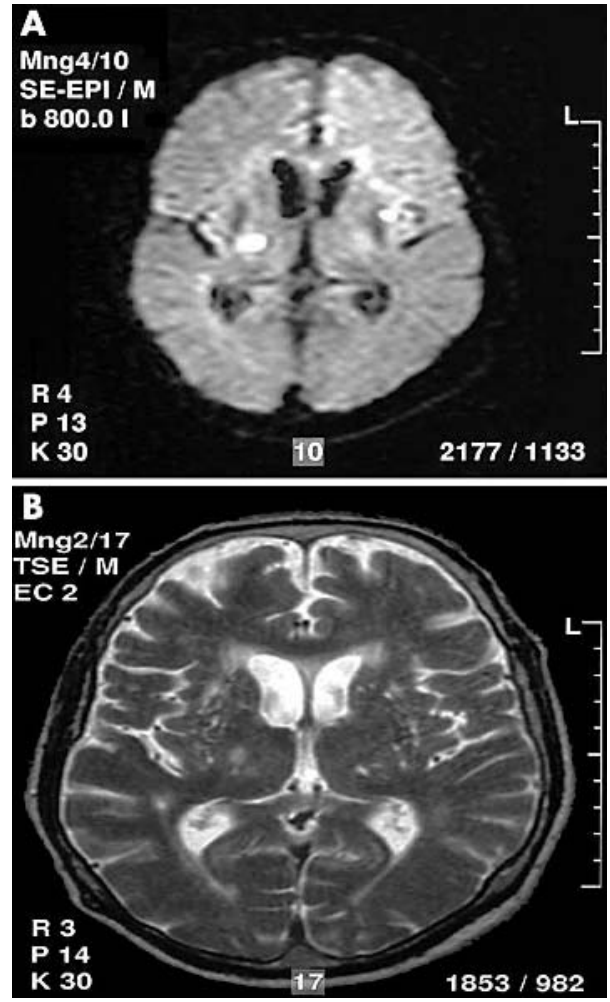

Figure 1 Axial diffusion weighted (A) and T2 weighted (B) magnetic resonance (MR) images of a patient presenting with a left sensorimotor hemisyndrome. Diffusion weighted MR imaging shows two small and hyperintense lesions which correspond to an acute symptomatic ischaemic lacunar infarct in the posterior limb of the right internal capsule and an asymptomatic acute ischaemic lacunar infarct in the left lentiform nucleus (type 1 multiple acute brain infarcts). The corresponding T2 weighted MR image (B) depicts the right capsular infarct as a hyperintense lesion, while the lenticular infarct is not definitely detectable.

history or by blood tests done during a hospital admission (venous plasma glucose concentration $\geqslant 7.0 \mathrm{mmol} / \mathrm{l}$ after an overnight fast on at least two separate occasions, and/or $\geqslant 11.1 \mathrm{mmol} / \mathrm{l}$ two hours after ingestion of $75 \mathrm{~g}$ oral glucose and on one other occasion during the two hour test);

- dyslipidaemia, defined by past medical history or blood samples taken within the first 48 hours after stroke onset: hypercholesterolaemia (total venous plasma cholesterol concentration $>5.0 \mathrm{mmol} / \mathrm{l}$ ), increased concentrations of low density lipoprotein (LDL) cholesterol (LDL cholesterol concentration $>3.0 \mathrm{mmol} / \mathrm{l}$ ), decreased concentrations of high density lipoprotein (HDL) cholesterol (HDL cholesterol concentration $<1.0 \mathrm{mmol} / \mathrm{l}$ ), increased ratio of total to HDL cholesterol $(>5)$, and hypertriglyceridaemia (triglyceride concentration $>1.6 \mathrm{mmol} / \mathrm{l}$ );

- history of coronary and peripheral artery disease;

- history of migraine with aura;

- history of amaurosis fugax (monocular blindness lasting $<24$ hours), retinal infarct (monocular blindness lasting $\geqslant 24$ hours), or transient ischaemic attack (TIA, neurological deficit lasting $<24$ hours).

The severity of the presenting neurological deficit was assessed by the National Institutes of Health stroke scale (NIHSS). ${ }^{7}$

Cardiological evaluation included a 12 lead electrocardiogram in all cases, while 24 hour Holter monitoring, transthoracic echocardiography, and transoesophageal echocardiography were carried out at the discretion of the treating physician.

Cerebral arteries were investigated using ultrasound, done by experienced sonographers with colour duplex scanners (Acuson XP 10 or Sequoia, Mountain View, California, USA). A detailed protocol of the ultrasound studies is published elsewhere. ${ }^{5}$

All patients underwent conventional magnetic resonance imaging (MRI) and DWI on a $1.5 \mathrm{~T}$ system with echo-planar imaging capability. Conventional MRI provided axial T1, T2, and proton density weighted images, and gadoliniumdiethylentriamine-pentaacetic acid enhanced Tl weighted images with a slice thickness of $5 \mathrm{~mm}$. DWI was obtained in the transverse plane with a single shot, echo-planar, spinecho pulse sequence with TR (time of repetition) of $6500 \mathrm{~ms}$, TE (time of echo) of $106 \mathrm{~ms}$, one excitation, and $b$ values $\left(1000 \mathrm{~s} / \mathrm{mm}^{2}\right)$. Diffusion gradients were applied simultaneously along the three axes $(x, y, z)$. The analysis of DWI scans was done by KB and RWB and in case of disagreement; every DWI was discussed individually in order to reach agreement.

\section{Classification of ischaemic strokes}

With the exception of lacunar strokes (see below), ischaemic strokes were classified according to the criteria of the TOAST study (trial of Org 10172 in acute stroke treatment). ${ }^{8}$ For correct TOAST classification, information from the DWI examination and on the presence of aortic plaques ${ }^{10}$ was considered.

Atherothrombotic strokes are characterised by presentation with ischaemic stroke and $>50 \%$ stenosis or occlusion of the supplying cerebral artery. Patients with aortic plaques of $\geqslant 4 \mathrm{~mm}$ diameter or mobile aortic thrombi located before the ostium of the left subclavian artery, but in whom no signs of cardioembolic and other determined aetiology of stroke were diagnosed at transoesophageal echocardiography, were also assumed to have atherothrombotic stroke. ${ }^{10}$

Cardioembolic strokes are characterised by presentation with an ischaemic stroke and a potential cardiac source of embolism.

Lacunar strokes are characterised by presentation with (1) one of the traditional clinical lacunar syndromes (pure motor hemiparesis, pure sensory syndrome, sensorimotor syndrome, ataxic hemiparesis, and dysarthria-clumsy hand syndrome ${ }^{1-14}$, and (2) either normal brain computed tomography/MRI or an appropriate subcortical or brain stem lesion of diameter $<1.5 \mathrm{~cm}$. Patients fulfilling the abovementioned clinical and neuroradiological criteria, in whom an atherothrombotic, cardioembolic, or other determined stroke aetiology was found, were classified as suffering from nonSAD lacunar strokes. ${ }^{5}$

Strokes with another determined aetiology refer to ischaemic strokes caused by arterial dissection, fibromuscular dysplasia, vasculitis, haematological disorder, migraine, and other rare forms of stroke.

Strokes of undetermined aetiology are ischaemic strokes where the underlying cause during diagnostic work up remains undetermined, or where there are multiple possible causes of stroke or an incomplete evaluation.

\section{Statistical analysis}

Statistical analysis was carried out with the "Systat" software package (Evanston, Illinois, USA). Differences between both types of MABI were compared by non-parametric analysis of variance (Mann-Whitney $U$ test). Two sided $p$ values of less than 0.05 were considered significant. 

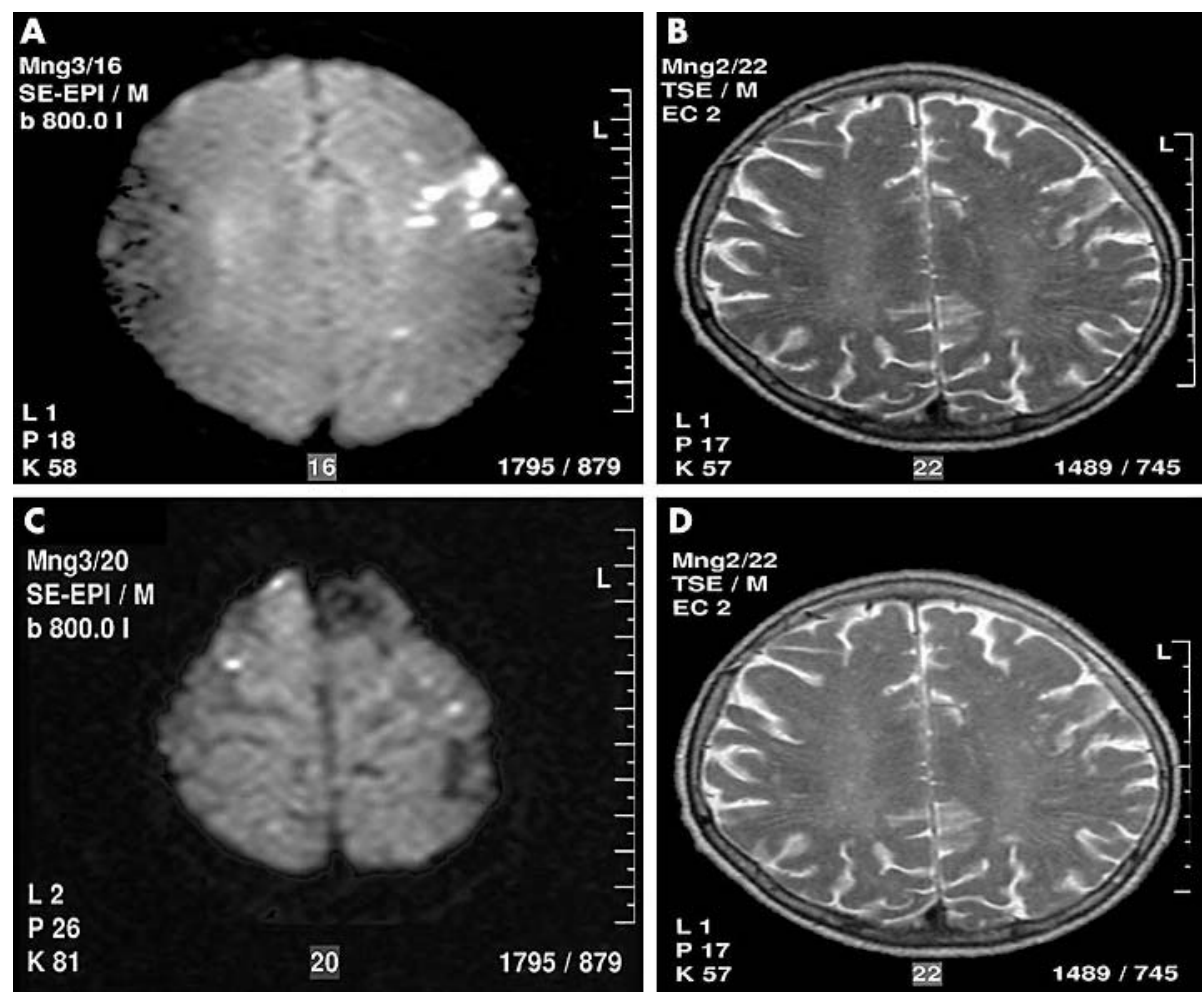

Figure 2 Axial diffusion weighted ( $A, C)$ and T2 weighted (B, D) magnetic resonance (MR) images of a patient presenting with motor dysphasia, dysarthria, and mild right brachiofacial hemiparesis. Diffusion weighted MR images show multiple acute (hyperintense) symptomatic infarcts with cortical-subcortical location in the left frontal lobe (A) and multiple acute, asymptomatic cortical infarcts in both hemispheres (C; type 2 multiple acute brain infarcts). The corresponding T2 weighted images show just the hyperintense left frontal infarct.

\section{RESULTS}

We evaluated 182 white patients with their first ischaemic stroke (115 men, 67 women). Their mean (SD) age was 62 (15) years (range 19 to 90). Twenty four hour Holter monitoring was carried out in $75(41 \%)$, transthoracic echocardiography in $118(65 \%)$, and transoesophageal echocardiography in 80 (44\%). The aetiology of the ischaemic strokes in all the patients studied, classified according to the modified TOAST criteria, is shown in table 1 , and the presenting characteristics of patients with the various MABI types are shown in table 2 .

MABI were found in 29 of 182 patients (16\%), including type $1 \mathrm{MABI}$ in 21 of 72 cases $(29 \%)$ with lacunar stroke, and type 2 MABI in eight of 110 cases (7\%) with non-lacunar stroke. NIHSS scores were significantly lower in MABI type 1. Other characteristics were equally present in both groups.

In nine of 21 type 1 MABI patients (43\%) another possible aetiology in addition to SAD was detected as a cause of stroke, and in three of eight type 2 MABI patients (38\%) (table 1).

All MABI patients with aortic plaques $\geqslant 4 \mathrm{~mm}(\mathrm{n}=3)$ or a cardiac source of embolism $(n=6)$ were treated with oral anticoagulants. This regimen was also prescribed for an 84 year old patient who presented with atrial fibrillation, a $70 \%$ carotid stenosis, a homolateral capsular stroke, and acute infarcts in the territories of the opposite middle cerebral and superior cerebellar arteries (type $1 \mathrm{MABI}$ ), who did not undergo carotid surgery because the stenosis was assumed to

Table 1 The aetiology of stroke and the presence of multiple acute brain infarcts (MABI) in 72 patients with lacunar stroke (type $1 \mathrm{MABI}$ ) and 110 patients with non-lacunar stroke (type $2 \mathrm{MABI}$ ) on diffusion weighted magnetic resonance imaging

\begin{tabular}{|c|c|c|c|c|}
\hline \multirow[b]{2}{*}{ Aetiology of stroke } & \multicolumn{2}{|c|}{ Type $1 \mathrm{MABI}(\mathrm{n}=72)$} & \multicolumn{2}{|c|}{ Type 2 MABI $(n=110)$} \\
\hline & Yes $(n=21)$ & No $(n=51)$ & Yes $(n=8)$ & No $(n=102)$ \\
\hline $\begin{array}{l}\text { Atherothrombosis } \\
\text { Large artery disease } \\
\text { Aortic plaques } \geqslant 4 \mathrm{~mm} \\
\text { Cardiac embolism } \\
\text { Other determined } \\
\text { Small artery disease } \\
\text { Undetermined }\end{array}$ & $\begin{array}{l}2(10) \\
0 \\
2(10) \\
4(19)^{*} \\
3(14) \dagger \\
0 \\
12(57)\end{array}$ & $\begin{array}{l}25(49) \\
4(8) \\
11(22) \\
4(8) \\
1(2) \\
21(41) \\
0\end{array}$ & $\begin{array}{l}1(12.5) \\
0 \\
1(12.5) \\
2(25.0) \\
0 \\
0 \\
5(62.5)\end{array}$ & $\begin{array}{l}29(28) \\
21(21) \\
8(8) \\
26(25) \\
6(6) \\
0 \\
41(40)\end{array}$ \\
\hline \multicolumn{5}{|c|}{$\begin{array}{l}\text { Values are } n(\%) \text {. } \\
\text { *Patients had atrial fibrillation }(n=2) \text {, an artificial heart valve }(n=1) \text {, and a patent foramen ovale with atrial septal } \\
\text { aneurysm }(n=1) \text {. } \\
\text { tOne patient each had an extracranial vertebral artery dissection extending into the intracranial segment, an } \\
\text { amyloid angiopathy, and a vasculitis. }\end{array}$} \\
\hline
\end{tabular}


Table 2 Presenting characteristics in 29 patients with multiple acute brain infarcts on diffusion weighted magnetic resonance imaging

\begin{tabular}{lll}
\hline Characteristic & $\begin{array}{l}\text { Type 1 MABI } \\
(\mathbf{n}=21)\end{array}$ & $\begin{array}{l}\text { Type 2 MABI } \\
(\mathbf{n}=8)\end{array}$ \\
\hline Age (years) (mean (SD)) & $62(15)$ & $58(16)$ \\
Male & $12(57)$ & $5(63)$ \\
Atrial fibrillation & $2(10)$ & $1(13)$ \\
Smoker & $11(52)$ & $2(25)$ \\
$\quad$ Current/previous & $5(24) / 6(29)$ & $2(25) / 0(0)$ \\
Hypertension & $9(43)$ & $2(25)$ \\
Diabetes mellitus & $4(19)$ & $1(13)$ \\
Total cholesterol $>5$ mmol/I & $14(67)$ & $5(63)$ \\
High density lipoprotein $<1$ mmol// & $2(10)$ & $1(13)$ \\
Low density lipoprotein $>3$ mmol/I & $12(57)$ & $3(38)$ \\
Total cholesterol/high density & & \\
lipoprotein $>5$ & $3(14)$ & $2(25)$ \\
Triglycerides $>1.68$ mmol/I & $7(33)$ & $3(38)$ \\
History of coronary artery disease & $3(14)$ & $2(25)$ \\
Peripheral artery disease & $2(10)$ & 0 \\
Amaurosis fugax and retinal infarct & 0 & 0 \\
Transient ischaemic attack & $4(19)$ & 0 \\
NIHSS score (mean (SD)) & $3.0(1.5)^{*}$ & $8.8(4.5)^{*}$ \\
\hline
\end{tabular}

Values are $\mathrm{n}(\%)$ unless stated otherwise. ${ }^{*} \mathrm{p}<0.001$.

MABI, multiple acute brain infarcts; NIHSS, National Institutes of Health stroke scale.

be asymptomatic. The three type 1 MABI patients with signs of a possible stroke aetiology other than $\mathrm{SAD}$ received aspirin, as well as the remaining 12 of 21 type 1 MABI patients without signs of any other determined stroke aetiology apart from SAD. Additionally, in the MABI type 2 patient group of five patients with stroke of undetermined aetiology, aspirin was chosen as secondary prevention.

\section{DISCUSSION}

In this study the prevalence of type l MABI was 29\% in patients with lacunar stroke, and the prevalence of both MABI types was $16 \%$ in all patients. One series reported $16 \%$ for MABI type $1,{ }^{4}$ while other investigators reported MABI in $17-29 \%$ of patients, without distinguishing between MABI type 1 and type $2 .{ }^{15-17}$ The results of the present study do not differ markedly from those already reported.

Detection of type 1 MABI in patients with lacunar stroke suggests that SAD is an unlikely aetiology. ${ }^{4}$ In fact, a recent study found a possible stroke aetiology other than SAD in eight of 10 type 1 MABI patients. ${ }^{4}$ The aetiologies included cardiac embolism in four cases, atherothrombosis in three, and cocaine associated vasculopathy in one. ${ }^{4}$ In the present study, $43 \%$ of type 1 MABI cases showed a potential cause of stroke other than SAD. It is unclear why there was such a difference in diagnosed stroke causes between the two studies. Generally, detection of the underlying aetiology of a stroke depends on the extent of the diagnostic work up, but the investigators did not report the type or the number of ancillary studies carried out, thus preventing any comparisons of the diagnostic work up. Conversely, in the present study more patients had transoesophageal echocardiography $(44 \% \vee 5 \%)$, which may explain the greater number of cases in which potential aortic sources of embolism were identified. However, the present MABI patients were on average five years younger than those investigated in Ay's study. ${ }^{4}$ It is well known that the prevalence of atherosclerosis and atrial fibrillation increases with age. ${ }^{18} 19$

The data from this and previous studies ${ }^{4}{ }^{16}$ suggest that the detection of MABI in patients with lacunar stroke should be followed by an intensive search for a stroke mechanism other than small artery disease. The diagnostic gain of detecting type 1 MABI depends, however, on the extent of the diagnostic work up carried out before doing the DWI studies and will thus vary from centre to centre and among different physicians. It is also important to note that different causes of MABI-such as cardiac embolism, vasculitis, or coagulopathy-have not yet been shown to produce a specific pattern of brain infarcts.

In patients presenting with lacunar stroke and a possible cardiac source of embolism it is unclear whether the lacune results from SAD or embolism. However, the presence of MABI supports cardiac embolism as mechanism of stroke. Consequently, all type I MABI patients who also showed signs of a cardiac source of embolism were treated with warfarin. Aortic plaques located before the origin of the left subclavian artery and with a diameter of $4 \mathrm{~mm}$ or more (grade IV) are a risk factor for recurrent ischaemic stroke, ${ }^{10} 20$ especially the non-calcified grade IV plaques. ${ }^{21}$ The results of a prospective, non-randomised, and small scale study suggest that stroke prevention is more efficient with warfarin than with aspirin or ticlopidine. ${ }^{22}$ Correspondingly, the European Stroke Initiative (EUSI) guidelines recommend warfarin in aortic plaques with a level IV grade of evidence. ${ }^{23}$ We thus assumed that all type 1 and type 2 MABI patients with aortic grade IV plaques and no other possible cause of stroke had suffered from aortic embolisation to the brain and treated them with warfarin.

The prevalence of type 2 MABI was $7 \%$ in our patients with non-lacunar stroke. To our knowledge, no other study has reported such data. As mentioned above, three of eight type 2 MABI patients with a stroke aetiology that included cardiac and aortic sources of embolism were treated with warfarin. Thus the detection of type 2 MABI improved diagnostic confidence and guided stroke prevention in only a few patients. Such a weak diagnostic gain was also found in a recent study which used DWI to examine 107 patients with $\geqslant 70 \%$ carotid stenosis assumed to be symptomatic. ${ }^{24} \mathrm{MABI}$ located in both cerebral hemispheres were detected in two patients who were found to have atrial fibrillation on subsequent 24 hour Holter monitoring. Thus the carotid stenoses were no longer considered symptomatic, and no carotid endarterectomy or stenting was carried out.

Twelve of 21 type 1 MABI cases with no signs of a stroke aetiology other than SAD, and five of eight type 2 MABI patients with strokes of undetermined aetiology were treated with oral aspirin, but as cerebral embolism might be the underlying pathomechanism, a controlled stroke prevention study comparing different antithrombotic drugs in these patients is necessary.

The limitation of the MABI concept is that the it remains ambiguous whether or not multiple infarcts occur simultaneously. In acute ischaemic stroke, the apparent diffusion coefficient (ADC) of water decreases, which contributes to the increased signal on DWI. ${ }^{25-27}$ In the subacute phase-that is, after about 7-14 days-the reduced ADC values return to baseline. ${ }^{28}$ Because acute and subacute lesions appear hyperintense on DWI, we cannot exclude the possibility that consecutive instead of simultaneous ischaemic episodes occurred during this short period. Furthermore, a lacunar stroke could theoretically activate the coagulation system in patients with small artery disease and cause further lacunar infarcts and MABI. To our best knowledge, however, no study has investigated this issue.

In the present study information that is not included in the TOAST criteria was used for the aetiological classification of ischaemic strokes. Symptomatic brain infarcts associated with grade IV aortic plaques as the only possible stroke mechanism were classified as atherothrombotic strokes. ${ }^{1021}$ Furthermore, DWI findings were used to define the mechanism of stroke and the antithrombotic treatment. We 
believe that it is useful to go beyond the TOAST criteria under certain circumstances.

Diffusion weighted MRI allows the detection of an acute brain infarct at a very early stage, as well as new brain infarcts located within chronic lesions, ${ }^{1-3} 29$ and possibly the determination of the underlying cause of stroke by assessing the pattern of early DWI lesions. ${ }^{30}$ Our study suggests that detection of type l MABI increases diagnostic confidence and the possibilities of stroke prevention in patients with lacunar stroke. Further studies are necessary to examine different stroke prevention regimens in MABI patients without a determined cause of stroke other than SAD.

\section{Authors' affiliations}

V Caso, K Budak, D Georgiadis, R W Baumgartner, Department of Neurology, University Hospital of Zürich, Zürich, Switzerland B Schuknecht, Department of Neuroradiology, University Hospital of Zürich

Competing interests: none declared

\section{REFERENCES}

1 Moseley ME, Kucharczyk J, Mintorovitch J, et al. Diffusion-weighted MR imaging of acute stroke: correlation with T2-weighted and magnetic susceptibility-enhanced MR imaging in cats. Am J Neuroradiol 1990;11:423-9.

2 Warach S, Chien D, Li W, et al. Fast magnetic resonance diffusion-weighted imaging of acute human stroke. Neurology 1992;42:1717-23.

3 Lutsep HL, Albers GW, DeCrespigny A, et al. Clinical utility of diffusionweighted magnetic resonance imaging in the assessment of ischemic stroke. Ann Neurol 1997:41:574-80.

4 Ay $\mathrm{H}$, Oliveira-Filho J, Buonanno $\mathrm{F}$, et al. Diffusion-weighted imaging identifies a subset of lacunar infarction associated with embolic source. Stroke 1999;30:2644-50.

5 Baumgartner RW, Sidler C, Mosso M, et al. Ischemic lacunar stroke in patients with and without other potential mechanism than small artery disease. Stroke 2003;34:653-9.

6 Kawachi I, Colditz GA, Stampfer MJ, et al. Smoking cessation and decreased risk of stroke in women. JAMA 1993;269:232-6.

7 Wityk RJ, Pessin MS, Kaplan RF, et al. Serial assessment of acute stroke using the NIH stroke scale. Stroke 1994;25:362-5.

8 Gordon DL, Bendixen BH, Adams HP, et al. Interphysician agreement in the diagnosis of subtypes of acute ischemic stroke: implications for clinical trials. Neurology 1993;43:1021-7.

9 Adams HP, Bendixen BH, Kappelle $\sqcup$, et al. Classification of subtype of acute ischemic stroke. Definitions for use in a multicenter clinical trial. TOAST. Trial of Org 10172 in Acute Stroke Treatment. Stroke 1993;24:35-41.
10 Amarenco $\mathbf{P}$, Cohen $A$, Tzourio $C$, et al. Atherosclerotic disease of the aortic arch and the risk of ischemic stroke. N Engl J Med 1994;331:1474-9.

11 Fisher CM. Lacunes: small, deep cerebral infarcts. Neurology 1965:15:774-84.

12 Fisher CM, Cole M. Homolateral ataxia and crural paresis: a vascular syndrome. J Neurol Neurosurg Psychiatry 1965;28:48-55.

13 Fisher CM, Curry HB. Pure motor hemiplegia from vascular origin. Arch Neurol 1965:13:30-44.

14 Fisher CM. A lacunar stroke: the dysarthria-clumsy hand syndrome. Neurology 1967;17:614-17.

15 Baird AE, Loevblad KO, Schlaug G, et al. Multiple acute stroke syndrome. Marker of embolic disease? Neurology 2000;54:674-8.

16 Roh J-K, Kang D-W, Lee S-H, et al. Significance of acute multiple brain infarction on diffusion-weighted imaging. Stroke 2000;31:688-94.

17 Takahashi K, Kobayashi S, Matui R, et al. The differences of clinical parameters between small multiple ischemic lesions and single lesion detected by diffusion-weighted MRI. Acta Neurol Scand 2002;106:24-9.

18 Agmon Y, Khandheria BK, Meissner I, et al. Association of atrial fibrillation and aortic atherosclerosis: a population-based study. Mayo Clin Proc 2001;76:252-9.

19 Feinberg WM, Blackshear JL, Laupacis A, et al. Prevalence, age distribution, and gender of patients with atrial fibrillation. Analysis and implications. Arch Intern Med 1995;155:469-73.

20 The French Study of Aortic Plaques in Stroke Study Group. Atherosclerotic disease of the aortic arch as a risk factor for recurrent ischemic stroke. N Engl J Med 1996;334:1216-21.

21 Cohen A, Tzourio C, Bertrand B, et al. Aortic plaque morphology and vascular events. Circulation 1997;96:3838-41

22 Ferrari E, Vidal R, Chevallier T, et al. Atherosclerosis of the thoracic aorta and aortic debris as a marker of poor prognosis: benefit of oral anticoagulation. J Am Coll Cardiol 1999;33:1317-22.

23 Leys D, Kwiecinski H, Bogousslavsky J, et al. Part 2. Prevention. Cerebrovasc Dis 2004;17(suppl 2):15-29.

24 Kastrup A, Schulz JB, Mader I, et al. Diffusion-weighted MRI in patients with symptomatic carotid artery disease. J Neurol 2002;249:1 168-74.

25 Mintorovitch J, Moseley M, Chileuitt L, et al. Comparison of diffusion and T2weighted MRI for the early detection of cerebral ischemia and reperfusion in rats. Magn Reson Med 1991;18:39-50.

26 Benveniste H, Hedlund LV, Johnson GA. Mechanism of detection of acute cerebral ischemia in rats by diffusion-weighted magnetic resonance microscopy. Stroke 1992;23:746-54.

27 Hossmann K, Fischer M, Bockhorst K, et al. NMR imaging of the apparent diffusion coefficient (ADC) for the evaluation of metabolic suppression and recovery after prolonged cerebral ischemia. J Cereb Blood Flow Metab 1994; 14:723-31

28 Schlaug G, Siewert B, Benfield A, et al. Time course of the apparent diffusion coefficient $(A D C)$ abnormality in human stroke. Neurology 1997:49:113-19.

29 Warach S, Gaa J, Siewert B, et al. Acute human stroke studied by whole brain echo planar diffusion-weighted magnetic resonance imaging. Ann Neurol 1995;37:231-41.

30 Kang DW, Chalela JA, Ezzeddine MA, et al. Association of ischemic lesion patterns on early diffusion-weighted imaging with TOAST stroke subtypes. Arch Neurol 2003;60:1730-4 